\title{
FINANCIAMIENTO PARA INVESTIGACIONES EN INNOVACIÓN SOCIAL DE LA ACADEMIA LATINOAMERICANA: RETOS Y DESAFÍOS
}

\author{
Mario Enrique Vargas Sáenz* \\ https://orcid.org/0000-0002-1081-3190 \\ Cristina Escudero Pulgarín** \\ https://orcid.org/0000-0002-0002-864X
}

RECIBIDO: Mayo 2021 / ACEPTADO: Julio 2021 / PUBLICADO: Septiembre 2021

\begin{abstract}
Como citar: Vargas Sáenz, Mario; Escudero Pulgarín, Cristina. (2021). Financiamiento para investigaciones en Innovación Social de la Academia Latinoamericana: Retos y desafíos. Telos: revista de Estudios Interdisciplinarios en Ciencias Sociales, 23 (3), Venezuela. (Pp. 747-762).

DOI: www.doi.org/10.36390/telos233.15
\end{abstract}

\section{RESUMEN}

Reflexionar sobre los retos y desafíos que un tema tan actual pero tan complejo como la innovación social encuentra en las decisiones y financiamientos de investigación en las Universidades Latinoamericanas se constituye en propósito a la vez que en una oportunidad para convocar la reflexión y el compromiso de las partes interesadas. Si bien las innovaciones pueden tener un impacto en la forma en que las personas se relacionan entre sí, autores como Putnam resaltan que se involucran en la sociedad y construyen capital social y el valor social refleja efectos positivos para los individuos, las comunidades y para sociedad. Este valor social a nivel micro, como en el beneficio que una persona obtiene al comprar una nueva tecnología, un medicamento o incluso un viaje a la tienda de comestibles, y a nivel individual, es lo que una persona valora más que el dinero pagado, como una computadora o una receta por lo que puede ser verdaderamente transformador. Metodológicamente, en la revisión documental y con las respuestas obtenidas frente a la encuesta aplicada sobre el financiamiento de la investigación en innovación social en algunas Universidades Colombianas, Mexicanas y Peruanas, se evidencia la dificultad que enfrentan los investigadores en esta materia y la deuda que en aporte al conocimiento en Innovación Social aún tiene la academia latinoamericana pues la innovación social sigue habitando solamente los ámbitos de la extensión universitaria más no en el de la investigación, impidiendo una mayor creación de valor social y un mayor impacto en la transformación de su entorno desde el conocimiento con propósito. Al concluir, se señalará

\footnotetext{
* Director de EAFIT Social, coordinador de la Maestría en Gerencia de Empresas Sociales para la Innovación Social y el Desarrollo Local, director del grupo de Investigación GUIES en Innovación, Empresarismo y sostenibilidad, Categoría A, Colciencias. Correo electrónico: mvargas@eafit.edu.co
}

** Maestrante en la Maestría en Gerencia de Empresas Sociales para la Innovación Social y el Desarrollo local. Correo electrónico:
cescuder@eafit.edu.co 
Financiamiento para investigaciones en Innovación Social de la Academia Latinoamericana: Retos y desafíos

frente al momento actual de pandemia que enfrenta nuestra sociedad latinoamericana, cuáles podrán ser los retos y desafíos más urgentes para que nuestras universidades sigan siendo forjadoras de humanidad y sociedad.

Palabras clave: Innovación social; valor social; valor público; inversión en proyectos de innovación social; investigación universitaria.

\section{Funding for research in Social Innovation of the Latin American Academy: Challenges}

\section{ABSTRACT}

Reflecting on the challenges that a topic as current but complex as social innovation encounters in the decisions and funding of research in Latin American universities is a central objective and an opportunity to convene the reflection and commitment of stakeholders. While innovations can have impacted the way people relate to each other, authors such as Putnam emphasize that they engage in society and build social capital, and social value reflects positive effects for individuals, communities, and society. Social value can also be seen at the micro-level as in the benefit a person gets from buying new technology, a drug, or even a trip to the grocery store. At the individual level, social value is what a person values more than the money paid, such as a computer or a prescription. On a societal level, this can be truly transformative. Methodologically, in the documentary review and with the answers obtained from the survey applied on the financing of research in social innovation in some Universities of Colombia, Mexico, and Peru, it is evident the difficulty faced by researchers in this matter and the debt that in Contribution to Knowledge in Social Innovation still has the Latin American academy since social innovation continues to inhabit only the fields of university extension but not that of research, preventing a greater creation of social value and a greater impact on the transformation of its environment of knowing with purpose. In the end, it will be pointed out, due to the current pandemic facing our Latin American society, what may be the most urgent challenges for our universities to continue building humanity and society.

Keywords: Social innovation; social value; public value; investment in social innovation projects; university research.

\section{Introducción}

Reflexionar sobre los retos y desafíos que un tema tan actual pero tan complejo como la innovación social encuentra en las decisiones y financiamientos de investigación en las Universidades Latinoamericanas se constituye en una oportunidad para convocar la reflexión y el compromiso de las partes interesadas. Este interés acompaña el surgimiento de la presente investigación no solo por hacer parte de una dirección universitaria centrada misionalmente en la innovación social y la contribución de las capacidades universitarias en el debate y alternativas de solución de los desafíos actuales que enfrenta la sociedad, sino además por la trayectoria investigativa que en los últimos quince años se ha definido como línea estratégica en el grupo 
de investigación GUIES ${ }^{1}$, y en la cual quienes escriben han estado activos igualmente desde el origen de la Maestría en Gerencia de Empresas Sociales para la Innovación Social y el Desarrollo Local2.

En la revisión de los resultados obtenidos, al momento de indagar sobre los programas y proyectos sociales en los últimos cinco años y su respectiva inversión económica, en ocho Universidades Colombianas, seis Mexicanas y cinco Peruanas, tomadas como muestra, no solo por las experiencias de trabajo previo que cada una de ellas ha tenido con EAFIT, sino por su relevancia en las distintas geografías nacionales, se evidencia en la primera parte del artículo la dificultad que enfrentan los investigadores en torno a la innovación social y la deuda que, frente a un aporte consolidado y colegiado, pueda aportar al conocimiento en este campo tiene aún la academia latinoamericana, y particularmente porque estos temas declarados como de interés institucional no reflejan el apalancamiento directo en el orden presupuestal para que áreas e investigadores puedan avanzar en la construcción de bases sólidas sobre las cuales avanzar en temas que, a nuestro modo, aportan a la solución de las crisis y los problemas estructurales de las sociedades actuales, al menos en nuestro continente.

Interesa pues la pregunta tanto de la existencia en el portafolio de investigación universitario de proyectos de innovación social como las experiencias académicas, investigativas y personales de los autores sobre este tema y, si bien América Latina ha sido reconocida como un continente de la esperanza y de la innovación, no se tiene una verdadera escuela de innovación social latinoamericana, lo que permitirá aventurarse a concluir en el horizonte de la pregunta ¿qué se necesita para crear una escuela latinoamericana de innovación social incubada en la academia de dicho continente?

Pareciera que la Innovación Social fuera un lugar común en el lenguaje -como muchos otros conceptos, vgr. Innovación pública - pero cuando se indaga al interior de la práctica directiva y de gestión de la academia, aún hoy la innovación social se ve más en el campo de la extensión universitaria más no en el de la investigación, y mucho menos se encuentra apalancada directa y estructuralmente por recursos propios de las universidades, impidiendo una mayor creación de valor social y un mayor impacto en la transformación de su entorno desde el conocimiento con propósito. Esto explica también la precariedad de las redes de investigación entre académicos latinoamericanos y la creciente interrelación con académicos y centros de excelencia norteamericanos, europeos y asiáticos, como mecanismo de visibilidad y gestión de recursos para sus proyectos.

Sin el ánimo de marcar una conclusión definitiva, se actuó desde un enfoque metodológico que rastrea diferentes fuentes documentales para la construcción de marcos teóricos y conceptuales que den luz a la reflexión que acompañan este escrito, indaga actores y tomadores de decisiones representativos de las Instituciones de Educación Superior identificadas y revisa sus reportes oficiales y públicos. Basados en los primeros hallazgos que se detallan más adelante sobre la encuesta ${ }^{3}$ realizada a Directores Administrativos y Financieros e investigadores sociales de diecinueve (19) universidades colombianas, mexicanas y peruanas

\footnotetext{
${ }^{1}$ GUIES, Grupo de Investigación en Innovación, Emprendimiento y Sostenibilidad, Categoría A, Colciencias, adscrito a la Escuela de Administración de la Universidad EAFIT, Medellín, Colombia.

2 https://www.eafit.edu.co/programas-academicos/posgrado/maestria-en-gerencia-de-empresas-sociales-para-la-innovacion-social-y-eldesarrollo-local/Paginas/inicio.aspx

${ }^{3}$ https://bit.ly/3lryT22
} 
Financiamiento para investigaciones en Innovación Social de la Academia Latinoamericana: Retos y desafíos

de carácter público y privado sobre los recursos que cada institución destina para la realización de programas y proyectos de innovación social, se señalará frente al momento actual de pandemia y otros desafíos sociales que enfrentan nuestros países, cómo podrán constituirse algunos de los retos y desafíos más urgentes para que las universidades - vgr. las de la muestra investigativa, entre otras -, actúen colegiadamente como una sólida academia latinoamericana y que emprendan y fortalezcan, desde la investigación en innovación social, sus capacidades y recursos, así como sus redes e interacciones transdisciplinares, para cumplir uno de sus postulados éticos como lo es el de ser forjadoras de humanidad y sociedad.

\section{Tópicos de análisis}

Algo propio de las Universidades y del mundo académico es sin duda el asunto de la investigación, de la creación, consolidación, divulgación y transferencia del conocimiento; sin investigación no hay desarrollo, sin conocimiento no hay innovación. Por ello la lente y el enfoque metodológico que parte desde un diagnóstico rápido, como si fuera una especie de prueba ácida, de las inversiones reales que algunas universidades de los tres países involucrados como muestra de este estudio, diecisiete de México, Perú y Colombia, con el propósito de evidenciar no solo el peso específico de este porcentaje de inversión frente al presupuesto total de la investigación universitaria nacional, sino la relevancia que posee la innovación social en el portafolio de dichos proyectos de investigación así como la existencia real de un sistema de conocimiento que soporte y dé línea metodológica y conceptual a las mismas.

\section{Análisis fáctico}

Si bien las innovaciones pueden tener un impacto en la forma en que las personas se relacionan entre sí, se involucran en la sociedad y construyen capital social (Putnam, et al., 1994); (Putnam, 2000); (Coleman, 1988), y el valor social refleja efectos positivos para los individuos, las comunidades y para sociedad. El valor social también se puede ver a nivel micro, como en el beneficio que una persona obtiene al comprar una nueva tecnología, un medicamento o incluso un viaje a la tienda de comestibles. A nivel individual, el valor social es lo que una persona valora más que el dinero pagado, como una computadora o una receta. $\mathrm{A}$ nivel social, esto puede ser verdaderamente transformador. Aquellos que están interesados en los aspectos comerciales, como los capitalistas de riesgo, a menudo se enfocan en el valor económico, mirando a través de la lente del espíritu empresarial comercial (Acs, et al., 2012).

Se despende un primer tópico de análisis en torno a la pregunta de ¿cómo es concebida la innovación social en la práctica - léase, revisando las declaraciones oficiales y publicas - de la academia universitaria en América Latina?

Algunas respuestas emanan de declaraciones de organismos internacionales que han sido orientadores para este tipo de praxis. Es el caso del Foro Internacional de Alto Nivel para el Desarrollo Sostenible, propiciado por la UNESCO con motivo del Año de la Innovación, (Sanz, 2017) en Guanajuato (Mx) en donde ampliamente se describen las innovaciones que debieran 
tocar de lleno el ámbito misional de la academia en regiones y particularmente en nuestro continente.

Foro en el que son señaladas y puestas en relieve por la directora Unesco México del momento, Nuria Sanz, las principales consideraciones para que en la innovación:

Se interrogue sobre la apropiación social del conocimiento y de las tecnologías; sobre la aceptación social de las biotecnologías; sobre la naturaleza y el carácter de los contextos de cambio tecnológico; una innovación que ve en la biotecnología, en la bioindustria y en sus regulaciones, nuevas formas de bioeconomía que necesitan repensar sus culturas empresariales; una innovación que nos sirva para repensar la naturaleza social y no sólo política o económica de los tratados de libre comercio y sobre el papel de la cooperación internacional en ello; una innovación preocupada por el uso y apropiación local de tecnologías globales; una innovación apegada a una academia que provea de análisis rigurosos socialmente comprometidos, pertinentes a la diversidad de los contextos sociales y culturales, que se dote sistemáticamente de un ejercicio riguroso de evaluación independiente - tal vez un llamado esencial que acompaña el motivo de este escrito en la búsqueda de una oportunidad de desarrollo desde la academia latinoamericana-, una innovación preocupada por sus formas de creación y por la protección de su valor y de su patrimonio intangible; una innovación preocupada por los efectos, quizá perversos (o no) de las patentes, y su manera de proteger (o no) los derechos humanos fundamentales en sociedades democráticas; una innovación para repensar la empresa, para impulsar el pensamiento creativo de las corporaciones gracias al capital creativo de cada uno/a de sus empleados/as; una innovación que quiere desenmascarar ciertas formas de ecologismo que actúan ideológicamente; una innovación sincera que entiende que la universalidad de acceso a energías limpias es una de las metas más difíciles y lejanas de la humanidad; una innovación para la salud, para lo preventivo y lo terapéutico, preocupada por personas diversas funcionalmente con capacidades especiales físicas o intelectuales; una innovación que sabe que tiene que enfrentar desafíos éticos respecto al uso y aplicación de tecnologías emergentes, sin dejar de garantizar el desarrollo armónico de la libertad y dignidad humanas (Sanz, 2017).

Ya la misma UNESCO lo había incluido en su declaración sobre la ciencia y la utilización del conocimiento científico, en el marco de la Conferencia Mundial sobre la Ciencia en 1999, cuando señalaba literalmente acerca de la dimensión de la ciencia para el desarrollo como:

Hoy más que nunca, la ciencia y sus aplicaciones son indispensables para el desarrollo. Los gobiernos, a todos los niveles, y el sector privado, deberían apoyar más activamente, a través de los correspondientes programas de educación y de investigación, la puesta en marcha de una capacitación 
Financiamiento para investigaciones en Innovación Social de la Academia Latinoamericana: Retos y desafíos

científica y tecnológica realmente compartida, base indispensable para un desarrollo económico, social, cultural, así como racional desde el punto de vista medioambiental. Esto es particularmente urgente para los países en desarrollo. El desarrollo tecnológico debe reposar sobre bases científicas sólidas, y orientarse hacia unas formas de producción limpias y sin riesgos, una utilización de los recursos más eficiente, y unos productos más respetuosos con el medioambiente.

La ciencia y la tecnología deben también orientarse hacia unas mejores perspectivas de empleo, así como a una mejora de la competitividad y de la justicia social. Es conveniente aumentar las inversiones en ciencia y en tecnología, cuidando tanto la realización de dichos objetivos, como permitir un mejor conocimiento y protección de los recursos naturales, la biodiversidad y unos sistemas globales para el mantenimiento de la vida. El objetivo debe ser progresar hacia estrategias de desarrollo duradero, gracias a la integración de las dimensiones económica, social, cultural y medioambiental (UNESCO, 1999, p. 3).

\section{Análisis institucional}

Un segundo tópico de análisis que brota de este diagnóstico inicial es el de interrogarse para validar la existencia o no de una verdadera escuela de innovación social en américa latina y que la misma de existir, esté alimentada por la investigación, los proyectos y los diálogos de saberes sociales y comunitarios, estimulados por el quehacer misional de las casas de estudio de educación superior.

En este aspecto es preciso retomar el concepto de oportunidad y los ciclos de oportunidad. La oportunidad es un elemento clave tanto en el emprendimiento comercial como de la innovación social (Mair, 2006). Además, los académicos distinguen entre oportunidades comerciales y sociales (Austin, et al., 2006); (Robinson, 2006), identificando diferencias en la misión y el valor creado por cada una. Si bien la creación de valor comercial se trata de la creación de riqueza personal y / o de los accionistas (Austin, et al., 2006), la creación de valor social se trata de "resolver problemas sociales como generar ingresos para las personas económicamente desfavorecidas o entregar suministros médicos a áreas afectadas por la pobreza del mundo y requiere innovación al igual que la creación de valor económico en el sector comercial" (Corner \& Ho, 2010). La creación de valor social tiene que ver con el impacto social.

Si se entiende la oportunidad social como una posible solución empresarial para abordar un problema social, dicha solución debe generar valor social, que se define como "aquello que mejora el bienestar de la tierra y sus organismos vivos" (Brickson, 2007); (Félix, et al., 2017). En la realización de un emprendimiento social, como expresión de procesos de innovación social, la universidad tiene la intención explícita de generar mejoras sociales a través de la movilización y asignación inteligente de sus recursos. De hecho, las mejoras sociales afectan el contexto donde operan las organizaciones, que se benefician directamente gracias a las iniciativas sociales que ayudan a desarrollar.

A pesar de sus innegables logros, en los albores de este siglo, las naciones latinoamericanas todavía tienen un amplio abanico de necesidades sociales insatisfechas, gran 
parte de ellas develadas y agudizadas por las crisis sociales y sanitarias que ha traído el Covid 19 entre otros factores. Afortunadamente, en cada una de estas naciones, un gran número de universidades, organizaciones sociales y comunitarias y de empresas trabajan a diario para dar respuesta a estos desafíos y cuando consiguen hacerlo, crean valor para la sociedad, es decir, contribuyen a mejorarla. Se crea valor cada vez que se da respuesta a una necesidad insatisfecha, pero, de otro lado, aún se evidencia una constante desconexión entre dichos resultados privando de la oportunidad que escalar sus resultados y aprender de sus impactos.

Un intento de respuesta podría encontrarse en las tipologías de ránquines de investigadores que privilegian determinadas publicaciones y que, en algunos casos, los resultados de apropiación social como resultados de innovaciones sociales cocreados desde la academia, no son tan "reconocidos" por lo que se quedan en los anecdotarios y reportes domésticos de cada universidad. Consecuencia directa... ausencia de repositorios vivos de resultados de investigación y de innovación social que, a modo de nuevo truque o intercambio virtuoso, alimenten proyectos, propuestas investigativas y debates en las aulas y pasillos de nuestros claustros universitarios.

\section{Análisis de pertinencia}

Aparece entonces un tercer tópico de análisis relacionado con el contexto de los emprendimientos y las innovaciones sociales que desatan lógicas de sostenibilidad como las empresas sociales, en el que esta promesa necesariamente implica alcanzar resultados e impactos sociales deseables que no están siendo generados en forma espontánea por los mercados. La proposición de valor es la piedra fundacional de cualquier emprendimiento social, ya que determina su foco estratégico y tiene implicaciones profundas para sus estructuras, procesos y asignación de recursos. La proposición de valor debería indicar qué tipo de valor se está generando.

El valor social se ha presentado como la búsqueda del progreso social, mediante la remoción de barreras que dificultan la inclusión, la ayuda a aquellos temporalmente debilitados o que carecen de voz propia y la mitigación de efectos secundarios indeseables de la actividad económica. En contraposición a lo que ocurre con el Valor Económico, que es capturado en forma autónoma por sus beneficiarios y remunerado con sus propios recursos en un intercambio comercial, en este caso el emprendimiento social ayuda al beneficiario a obtener un valor que, por distintos motivos, habría estado fuera de su alcance, y dicho espíritu emprendedor acompaña la génesis y la vida de la universidad y de la academia aún hoy.

Al afirmar que un proyecto de innovación social combina la creación de valor económico con la de valor social, y que dicha relación también opera en la práctica investigativa académica, se está diciendo que por un lado crea valor que puede ser capturado y retribuido por los destinatarios (por ejemplo, clientes con gran poder adquisitivo, caso de los negocios inclusivo o recientemente de las empresas $\mathrm{BIC}$ ), y que al mismo tiempo crea valor socialmente deseable que no habría podido ser remunerado en forma autónoma por los beneficiarios (Austin, et al., 2006). Este hecho podría explicar porque algunas universidades no le dan tanto peso a proyectos e investigaciones de innovación social desde el punto de vista de los réditos o retornos financieros - de corto y mediano plazo - que dichas iniciativas entreguen al alma mater.

Si bien uno de los roles de la innovación social en la sociedad es el de la creación de valor social a través de la innovación y los intercambios mutuamente beneficiosos para resolver 
Financiamiento para investigaciones en Innovación Social de la Academia Latinoamericana: Retos y desafíos

problemas, es preciso reafirmar que dicha creación de valor social sucede directa y de forma constante y progresiva al abordar problemas que se identifican y pueden abordarse en el corto y mediano plazo, lugar natural de la praxis investigativa. Como cualquier empresa o sector exitoso y en crecimiento o desarrollo, lo mismo acontece en la academia de manera que el emprendimiento social puede crear oportunidades, aunque de manera indirecta (Acs, et al., 2012).

Este fenómeno ha dado origen a uno de los lugares de interrelación y conexión entre la investigación y la empresa con la sociedad como son las spin-off. Esto puede iniciar desde una pequeña empresa individual hasta un cambio social a gran escala (Acs, et al., 2012). Y acá una nueva pregunta a la Academia y a la Universidad latinoamericana, ¿estos retos y estos propósitos no coinciden casi literalmente con nuestras misiones y definiciones estratégicas de Universidad? ¿No pueden ser las spin-off alternativas de desarrollo sostenible y de impacto social de nuestros grupos de investigación?

Finalmente y a pesar del propósito de dichos resultados de carácter comercial o empresarial, la empresa social y su misión social sigue siendo la piedra angular de la organización matriz sin fines de lucro y es fundamental para su ética y ocupaciones, tal como lo ha manifestado en uno de sus textos Alter Kim, "Social Enterprise Models and Their Mission and Money Relationships" citado por Nicolls, 2006, en los que se afirma como dado que las propiedades de creación de valor se entrelazan en la empresa social, el propósito y la misión están indisolublemente ligados. De hecho, la interrelación entre Las actividades comerciales y los programas sociales están dictados por la empresa social. Modelos de empresas sociales propósito y relevancia para la misión de la organización matriz. Por tanto, un sistema de clasificación basada en la "orientación de la misión" proporciona un factor determinante del tipo de empresa social.

En la empresa social centrada en la misión, las actividades comerciales son fundamentales para la misión social de la organización matriz. Estas empresas sociales se crean para el propósito expreso de hacer avanzar la misión utilizando un modelo de autofinanciamiento. La creación de valor social, en cambio, consiste esencialmente en cambiar para bien las vidas de los individuos, mediante la consecución de objetivos socialmente deseables (Nicholls, 2006). Con esto queda marcada la ruta de satanizar los desarrollos e impactos comerciales y empresariales de la investigación como si fuese en contravía de la equidad social; por el contrario, se convierte en auxilio estratégico que alimenta y sostiene el espíritu y la práctica investigativa de nuestras universidades en la búsqueda e incesante de una verdad que arrope de dignidad los pueblos y sociedades por ellas impactados y acompañados.

\section{Resultados iniciales}

Indagadas por medios digitales y apoyados en tecnologías de información debido a la pandemia, se procedió a contactar diecinueve (19) universidades de carácter público y privado que cuentan con alianzas o relacionamiento con la Universidad EAFIT como parte de esta primera muestra incluyendo países como Colombia, México y Perú: 
Cuadro 1.

\begin{tabular}{|c|c|c|}
\hline \multirow{3}{*}{$\begin{array}{c}\text { Colombia } \\
\text { Universidad EAFIT } \\
\text { www.eafit.edu.co/social } \\
\end{array}$} & México & Perú \\
\hline & $\begin{array}{l}\text { Universidad de Guanajuato } \\
\text { https://www.ugto.mx/ }\end{array}$ & $\begin{array}{l}\text { Universidad del Pacífico } \\
\text { www.unipacifico.edu.co:8095/ } \\
\text { web3.0/institucional.jsp?opt=2 }\end{array}$ \\
\hline & & $8 \& \mathrm{opt} 2=\mathrm{pg}$ \\
\hline & & 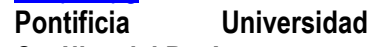 \\
\hline $\begin{array}{l}\text { Pontificia Universidad } \\
\text { Javeriana }\end{array}$ & $\begin{array}{l}\text { Universidad Autónoma de } \\
\text { Tamaulipas }\end{array}$ & $\begin{array}{l}\text { Católica del Perú } \\
\text { www.centrumthink.pucp.edu.p }\end{array}$ \\
\hline www.javeriana.edu.co/investig & www.uat.edu.mx/SitePages/princi & e/centros/centro-de- \\
\hline $\begin{array}{l}\text { acion/presentacion-de-la- } \\
\text { direccion-de-innovacion }\end{array}$ & pal.aspx & $\begin{array}{l}\text { investigacion-para-la- } \\
\text { sostenibilidad-y-la-innovacion- } \\
\text { social/ }\end{array}$ \\
\hline Universidad de La Sabana & & Universidad de Lima \\
\hline www.unisabana.edu.co/portal & Universidad Baja California & www.ulima.edu.pe/ceitex/agen \\
\hline $\begin{array}{l}\text { denoticias/al-dia/innovacion- } \\
\text { social/ }\end{array}$ & $\underline{\text { www.ubc.edu.mx/ }}$ & $\begin{array}{l}\text { da/coloquio-moda-innovacion- } \\
\text { artesania }\end{array}$ \\
\hline $\begin{array}{l}\text { Universidad ICESI } \\
\text { https://www.icesi.edu.co/launi }\end{array}$ & $\begin{array}{l}\text { Universidad Ciudad de Juárez } \\
\text { www.uacj.mx/VidaUniversitaria/in }\end{array}$ & $\begin{array}{l}\text { Universidad Privada del } \\
\text { Norte }\end{array}$ \\
\hline versidad/ & dex.html & $\begin{array}{l}\text { www.upn.edu.pe/search2?que } \\
r y=\text { social }+\end{array}$ \\
\hline Universidad CESA & $\begin{array}{l}\text { Universidad Autónoma del } \\
\text { Estado de México }\end{array}$ & Universidad San Marcos \\
\hline$\underline{\text { https://www.cesa.edu.co/ }}$ & $\begin{array}{l}\text { www.sev.uaemex.mx/index.php/s } \\
\text { ervicios/servicio-social }\end{array}$ & www.unmsm.edu.pe/ \\
\hline $\begin{array}{l}\text { Universidad del Norte } \\
\text { https://www.uninorte.edu.co/c } \\
\text { omunidad }\end{array}$ & $\begin{array}{l}\text { Universidad Nacional } \\
\text { Autónoma de México } \\
\text { www.unam. } m x /\end{array}$ & \\
\hline $\begin{array}{l}\text { Universidad de Los Andes } \\
\text { https://uniandes.edu.col }\end{array}$ & & \\
\hline $\begin{array}{l}\text { Universidad de La Guajira } \\
\text { https://www.uniguajira.edu.co/ }\end{array}$ & & \\
\hline$\frac{\text { centro-de-investigaciones- }}{\text { presentacion }}$ & & \\
\hline
\end{tabular}

Fuente: Elaboración propia

Con el propósito de obtener una prueba ácida, como se diría en la administración tradicional, que porcentaje de los fondos propios destinados a la investigación es asignado directamente a proyectos de innovación social. Las personas destinatarias de estas preguntas fueron diversas, entre directores administrativos, directores de investigación y algunos colegas investigadores que participan activamente desde sus grupos o cuerpos de investigación en estos temas de interés.

Los resultados son paradójicamente entre desconsoladores y alentadores.

En primer lugar, la cantidad de respuestas recibidas de las personas encuestadas no supero el $30 \%$ de la muestra. Revisados los programas directamente asociados en las universidades a las líneas de investigación en innovación social se describen: Diplomado en 
Financiamiento para investigaciones en Innovación Social de la Academia Latinoamericana: Retos y desafíos

empresa social, Universitarios para Trascender, Incubadora de proyectos de innovación social , Grupo de Innovación Social, Seminario Universitario de Emprendimiento Social, Administración Sostenible y Formación Integral en los Niveles Medio Superior y Superior en la Universidad Nacional Autónoma de México, Proyectos comunitarios, Reciclaje permanente, red de liderazgo juvenil y el voluntariado.

Cuando se preguntó en la encuesta, ¿Su universidad asigna recursos económicos para el desarrollo de los programas o proyectos de Innovación Social?, respondieron afirmativamente cinco (5) universidades y una declaro que no asignan este tipo de recursos.

De las diecinueve (19) universidades indagadas solamente dos (2) entregaron cifras concretas de recursos de caja asignados a la investigación. Una señala los siguientes recursos invertidos: COP\$1.682.500.000 en el 2015, COP\$1.414.700.000 en el 2016, COP\$269.370.760 en el 2017, COP\$838.000.000 en el 2018, COP\$930.000.000 en el 2019 y COP $\$ 420.000 .000$ en el 2020. La segunda menciona una cifra exacta, pero señala como estimado un millón de pesos mexicanos en financiamiento a proyectos de innovación social en los años 2015, 2016, $2017,2018,2019$ y 2020 respectivamente. De las demás, trece (13) de ellas declararon no tener los datos -algunas luego de hacer un tránsito interesante vía correo electrónico entre diferentes destinatarios de cada ateneo para identificar quien lo podría conocer - y las restantes dejaron solo en leído (como suele acontecer hoy día) anunciando la respuesta, pero cuando se elabora este artículo aún la respuesta no ha sido recibida. Hubo respuestas igualmente imprecisas en la que se señalaba que la persona que podía dar la información estaba trabajando desde la casa, no tenía acceso a las fuentes de información, o en algunos casos no estaba disponible...

Tal vez igualmente exista una ruptura en la forma en que le apostamos a la investigación en innovación social. Tal vez en algunos casos - lo conocimos extraoficialmente los asuntos de innovación social son bienvenidos en las prácticas de investigación universitarias y traen consigo los fondos necesarios para adelantar dichas investigaciones, es decir, si no solo traen la propuesta de investigación sino su financiador -en muchos casos el Estado- pero eso sí, sin la renuncia el "margen" o contribución líquida de caja de la investigación a las arcas universitarias. Se plantea entonces la duda si lo social es un lugar común o realmente hace parte del llamado misional, institucional y de responsabilidad de la gestión y dirección universitaria latinoamericana no solo en los asuntos de extensión -presencia más viva a través particularmente de los voluntariados universitarios, asuntos académicos y de inclusión -en algunos casos solo acontecen vía gratuidad o subsidio - pero en los asuntos investigativos brillan por su ausencia.

Sin embargo - y es la parte alentadora - las innovaciones sociales pueden tener un impacto en la forma en que las personas se relacionan entre sí, se involucran en la sociedad y construyen capital social (Putnam, et al., 1994); (Putnam, 2000); (Coleman, 1988), y el valor social refleja efectos positivos para los individuos, las comunidades y para la sociedad, no siendo la academia la excepción. De hecho, si dicho valor social de forma colectiva se puede ver también a nivel micro, como en el beneficio que una persona obtiene al comprar una nueva tecnología, un medicamento o incluso un viaje a la tienda de comestibles, o a nivel individual, en la medida en que el valor social es lo que una persona valora más que el dinero pagado, como una computadora o una receta. Imaginemos entonces a nivel social, lo que puede suceder cuando uno de los actores de mayor incidencia por su ética, objetividad y pertinencia es sin lugar 
a duda la Universidad... esto puede ser verdaderamente transformador... una verdadera revolución desde la investigación innovadora socialmente. Recientemente, algunas de las universidades consultadas a través de sus cuerpos o grupos de investigación reportan sus resultados e impactos asociados a la agenda 2030 o directamente a los ODS... un avance en la construcción de parámetros universales que permitan colegiar agendas de trabajo colectivo.

Aquellos que están interesados en los aspectos comerciales, como los capitalistas de riesgo, a menudo se enfocan en el valor económico, mirando a través de la lente del espíritu empresarial comercial (Acs, et al., 2012). Por otro lado, aquellos interesados en la creación de valor social pueden examinar la empresa a través del lente del emprendimiento social. Ambas lentes son apropiadas y la elección de una de ellas depende del interés o pregunta particular. De hecho, es totalmente posible considerar una empresa tanto en la riqueza generada como en el valor social de los bienes / servicios ofrecidos (Acs, et al., 2012). Esto ha permitido capitalizar la competencia investigativa y el talento y compromiso de muchos investigadores por parte de gobiernos y empresas para trascender y acompañar la transformación de las demandas y necesidades sociales en verdaderas y sostenibles oportunidades.

\section{Retos y desafíos}

Parte de los retos y desafíos estarán sintonizados con la innovación social como eje de investigación-acción de las Universidades y será un instrumento poderoso de creación de valor no solo social sino valor público. una innovación que sabe reconocer lecciones sociales y culturales en las mal llamadas formas de economía informal y que considera que son un capital social indiscutible (UNESCO, 2018).

La literatura sobre valores públicos distingue entre valores públicos, que son muchos por ejemplo, Van Wart, (1998); Bozeman, (2002); Bozeman, (2007); Jørgensen \& Bozeman, (2007); Meynhardt, (2009); Andersen, et al., (2012), crear valor público, definido como producir lo que el público valora, es bueno para el público, incluida la adición a la esfera pública, o ambos, según se evalúa en función de varios criterios de valor público Benington \& Moore, (2011); Stoker, (2006); Alford, Owen, (2008); Alford \& O’Flynn, (2009) y la esfera pública o ámbito público dentro del cual los valores públicos y los valores se desarrollan y desarrollan (Benington, 2013); (Bryson, et al., 2014).

Voces líderes en la literatura sobre valores públicos se centra en el nivel político 0 social escriben, entre otros asuntos que "Los valores públicos de una sociedad son aquellos que brindan un consenso normativo sobre: (1) los derechos, beneficios y prerrogativas a los que los ciudadanos deberían (y no deberían) tener; (2) las obligaciones de los ciudadanos con la sociedad, el estado y entre sí; y (3) los principios en los que se deben basar los gobiernos y las políticas" (Bozeman, 2007). Aunque los valores públicos en una democracia son típicamente cuestionados, se puede discernir un consenso relativo de constituciones, mandatos legislativos, políticas, revisiones de literatura, encuestas de opinión y otros (Bryson, et al., 2014).

Mark Moore, otra voz importante en la literatura se centra en los administradores públicos (Moore, 1995). Moore cree que los ciudadanos quieren de sus gobiernos una combinación de lo siguiente que, en conjunto, abarque el valor público: (1) público orientado al servicio de alto desempeño burocracias, (2) organizaciones públicas que son eficientes y efectivas para lograr los resultados sociales deseados, y (3) organizaciones públicas que operan de manera justa y equitativa, y conducen a condiciones justas y equitativas en la sociedad en 
Financiamiento para investigaciones en Innovación Social de la Academia Latinoamericana: Retos y desafíos

general (Bryson, et al., 2014). Meynhardt no es prescriptivo; tiene una base mucho más psicológica; y enfatiza más la interpretación de las esferas pública y privada (Bryson, et al., 2014). Los estudiosos de los valores públicos buscan en una variedad de fuentes evidencia de lo que el "público" es, quiere o es bueno para él (Bryson, et al., 2014). Los valores públicos definen un espíritu que a menudo se distingue del sector privado, por ejemplo, al centrarse en el interés público y el uso eficiente del dinero de los contribuyentes (Hofmann, et al., 2019).

El valor público es un valor para el público. El valor para el público es el resultado de evaluaciones sobre cómo las necesidades básicas de los individuos, los grupos y la sociedad en su conjunto se ven influidas en las relaciones que involucran al público. El valor público, entonces, es también valor del público, es decir, "extraído" de la experiencia del público. El público es una ficción operativa indispensable de la sociedad. Cualquier impacto en la experiencia compartida sobre la calidad de la relación entre el individuo y la sociedad puede describirse como creación de valor público. La creación de valor público se sitúa en las relaciones entre el individuo y la sociedad, fundamentadas en los individuos, constituidas por evaluaciones subjetivas frente a las necesidades básicas, activadas y realizadas en estados emocionales-motivacionales, y producidas y reproducidas en prácticas intensivas en experiencia (Meynhardt, 2009).

La creación de valor público desde la Universidad desplegando su capacidad investigativa, de creación y descubrimiento para la innovación social acontece también como el equilibrio de los valores combinados que son creados por diferentes resultados, más que como la producción efectiva de resultados independientes que no tienen que ser intercambiados (Cordella \& Paletti, 2019). Equilibrar los valores creados por diferentes resultados es una prescripción fundamental que la administración pública debe seguir para entregar valor público y evitar generar el descontento de los ciudadanos (Williams, et al., 2015); (Cordella \& Paletti, 2019) lo que no deja reparos a la necesidad de la colegialidad, rasgo incontrastable de la universidad en sus orígenes, pues la creación de valor público no solo se preocupa por satisfacer una sola necesidad colectiva a la vez (por ejemplo, un sistema de transporte público más eficiente), y con múltiples necesidades y expectativas que pueden competir 0 anularse entre sí (Alford \& Hughes, 2008); (Broussine, 2015), sino además con la capacidad de adaptarse a los cambios en las expectativas de valor (Cordella \& Paletti, 2019).

Estas consideraciones contrastan y alimentan la esperanza de la academia latinoamericana unida en un espíritu investigador desde la innovación social como creadora de valor público, dado el rol de la Universidad como bien público y de la Educación como valor público y social. Tal como lo mencionaba Meynhardt, "El valor público es lo que impacta en los valores sobre el 'público'" y la Universidad trasciende la formación con su impacto a cada una de las capas que conforman la sociedad en las que se inscribe y participa. El impacto no solo implica una apreciación positiva, sino, en primer lugar, algo, positivo o negativo, que importa para los valores de las personas sobre su relación percibida con "el público". Desde este punto de vista, el valor público no se limita a un determinado sistema de valores (cualquier sistema religioso o constitución, derechos humanos, democracia, etc.) (Meynhardt, 2009).

La creación de valor público es un proceso que se compara con la evaluación psicológica a nivel individual y grupal. En esta noción amplia, producir "hechos" no está necesariamente correlacionado con la creación de valor. Si un valor no está en la mente de las 
personas, no es "real" (Meynhardt, 2009). La creación de valor público implica una acción gerencial para equilibrar múltiples objetivos de la creación de valor público porque hay que intercambiarlos (Benington, 2013); (Hartley, et al., 2016); (Cordella \& Paletti, 2019). Este es un claro llamado a la cohesión social, a la postura transdisciplinar e interinstitucional que propicie conversaciones entre investigadores, sus proyectos y resultados, logros y fracasos - también los hay y más en el ámbito de la innovación social que adolece de data consistente en algunas geografías de nuestra región - y encuentre en dichas capacidades y experiencias un sustrato ideal para avanzar.

En consecuencia, para producir y entregar valor público, no es suficiente satisfacer una necesidad específica, sino que es necesario orquestar la forma en que la configuración de producción impacta en otras necesidades y valores a lo largo del tiempo, y adaptarse cuando estas necesidades cambian (Alford \& Hughes, 2008); (Bryson, et al., 2014); (Cordella \& Paletti, 2018); (Reynaers \& Graaf, 2014); (Stoker, 2006). Esto implica comenzar con un poco de ingeniería para entender profundamente el problema en la lógica de dinámica de sistemas. Segundo, llevar a cabo algo de psicología para entender profundamente los actores humanos que están en ese sistema. Tercero, plantear estrategias de gobernanza que aprovechan el conocimiento que existe en esos actores. Por último, en vez de copiar soluciones, permitir que las soluciones emerjan del mismo sistema para lograr los resultados esperados (Sanz, 2018).

\section{Conclusiones}

Lo que se había observado empíricamente desde lo que se había llamado el efecto Mariposa, es que existen sistemas que pueden desplegar un comportamiento impredecible (lo que no quiere decir «no sujeto a leyes»): pequeñas diferencias en una sola variable tienen efectos profundos en la historia posterior del sistema (Sánchez, 2009). Hoy tenemos sistemas compuestos y complejos con productos, intereses y capacidades que exceden la dificultad de la predicción y, al contrario, anticipan genuinamente los albores de una nueva sociedad en la que cada uno de sus integrantes valore y valide la contribución que la investigación científica y aplicada ofrecen en cada uno de sus resultados desde la práctica participativa consciente.

Algunos países latinoamericanos, entre ellos los tres seleccionados como muestra para esta investigación, han demostrado que es posible luchar eficazmente contra la pobreza y los grandes desafíos globales que han generado inequidad y perdida dignidad en los habitantes de sus propios territorios. En esta gesta las Universidades han desempeñado un rol importante, pero que en muchos casos no ha trascendido. Sus hallazgos, estudios e investigaciones se han quedado almacenadas y perdidas en sólidos repositorios y soberbios anaqueles.

Esa responsabilidad social y pública, con lo público y con el público, de la Universidad a veces se ha vuelto paisaje... no ha trascendido, no ha generado el efecto ni el impacto esperado ni debido. El mecanismo para llegar a esta conclusión, léase la aplicación de una encuesta a diecinueve universidades latinoamericanas permite predecir un camino aún muy complejo por recorrer para aquellos quienes ven en la innovación social no solo una apuesta concreta en la generación de valor social y público en cada uno de sus territorios sino especialmente en ver a través de ella y de sus resultados oportunidades de investigación, de construcción de conocimiento, de apropiación del mismo y de cambio social.

Por ello, proponer una reflexión sobre cómo la academia latinoamericana que habla de innovación social debe materializar su compromiso con recursos, y recursos líquidos, a las 
Financiamiento para investigaciones en Innovación Social de la Academia Latinoamericana: Retos y desafíos

investigaciones y las apuestas que en materia de innovación social en nuestras universidades ya están generando valor público y que desde ahí son eje de transformación desde la educación de la sociedad. Estamos afirmando que toda inversión económica en proyectos de investigación y su ejercicio de divulgación de sus resultados constituye una verdadera y nueva pedagogía de la esperanza y de la hermandad solidaria, particularmente en momentos como los que América Latina enfrenta cotidianamente de pandemias en salud (Covid-19), en valores (desafíos y crisis sociales e institucionales) y, tal vez la más grave que es la pandemia de la indiferencia.

\section{Referencias Bibliograficas}

Acs, Zoltan; Audretsch, David; Braunerhjelm, Pontus. (2012). Growth and entrepreneurship. Small Business Economics, 39(2), Países Bajos. (Pp.213-245). DOI: https://doi.org/10.1007/s11187-010-9307-2

Alford, John; Hughes, Owen. (2008). Public Value Pragmatism as the Next Phase of Public Management. The American Review of Public Administration, 38(2), Estados Unidos. (Pp.130-148). DOI: https://doi.org/10.1177/0275074008314203

Alford, John; O'Flynn, Janine. (2009). Making Sense of Public Value: Concepts, Critiques and Emergent Meanings. International Journal of Public Administration, 32(3-4), Estados Unidos. (Pp.171-191). DOI: https://doi.org/10.1080/01900690902732731

Andersen, Lykke; Busch, Jonah; Curran, Elizabeth; Ledezma, Juan; Mayorga, Joaquin; Bellier, Mélissa. (2012). Environmental and socio-economic consequences of forest carbon payments in Bolivia: Results of the OSIRIS-Bolivia model. Institute for Advanced Development Studies, Bolivia.

(Pp.1-35). https://www.inesad.edu.bo/pdf/wp2012/wp02_2012.pdf

Austin, James; Stevenson, Howard; Wei-Skillern, Jane. (2006). Social and Commercial Entrepreneurship: Same, Different, or Both? Entrepreneurship Theory and Practice, 30(1), Estados Unidos. (Pp.1-22). DOI: https://doi.org/10.1111/j.15406520.2006.00107.x

Benington, John. (2013). From Private Choice to Public Value. Warwick Business School, Institute of Governance and Public Management (IGPM), Reino Unido (Pp.1-36). https://www.researchgate.net/publication/252055942_From_Private_Choice to_Public Value

Benington, John; Moore, Mark. (2011). Public Value: Theory and Practice. Macmillan International. Estados Unidos.

Bozeman, Barry. (2002). Public-value failure: When efficient markets may not do. Public Administration Review, 62(2). Reino Unido. (Pp.145-161). https://www.jstor.org/stable/3109898

Bozeman, Barry. (2007). Public Values and Public Interes: Counterbalancing economic individualism. Georgetown University Press. Estados Unidos.

Brickson, Shelley. (2007). Organizational Identity Orientation: The Genesis of the Role of the Firm and Distinct Forms of Social Value. The Academy of Management Review, 32(3). Estados Unidos. https://doi.org/10.5465/amr.2007.25275679 
Broussine, Mike. (2015). Public Leadership. En Bovaird, Tony; Löffler, Elke (Eds.). Public Management and Governance (3rd ed.). Routledge. Inglaterra. DOI: https://doi.org/10.4324/9781315693279

Bryson, John; Crosby, Barbara; Bloomberg, Laura. (2014). Public Value Governance: Moving Beyond Traditional Public Administration and the New Public Management. Public Administration Review, 74(4). Estados Unidos. (Pp.445-456). DOI: https://doi.org/10.1111/puar.12238

Coleman, James. (1988). Social Capital in the Creation of Human Capital. American Journal of Sociology, 94. Estados Unidos. (Pp.95-120). https://www.jstor.org/stable/2780243

Cordella, Antonio; Paletti, Andrea. (2018). ICTs and value creation in public sector: manufacturing logic vs service logic. The London School of Economics and Political Science, 23 (2). Reino Unido. (Pp.125-141). DOI: https://doi.org/10.3233/IP-170061

Cordella, Antonio; Paletti, Andrea. (2019). Government as a platform, orchestration, and public value creation: The Italian case. Government Information Quarterly, 36(4). Reino Unido. (Pp.1-13). DOI: https://doi.org/10.1016/i.giq.2019.101409

Corner, Patricia; Ho, Marcus. (2010). How Opportunities Develop in Social Entrepreneurship. Entrepreneurship Theory and Practice, 34(4). Nueva Zelanda. (Pp.1-25). DOI: https://doi.org/10.1111/i.1540-6520.2010.00382.x

Félix, Mónica; Husted, Bryan; Aigner, Dennis. (2017). Opportunity discovery and creation in social entrepreneurship: An exploratory study in Mexico. Journal of Business $\begin{array}{llll}\text { Research, } 81 . \quad \text { Estados Unidos. (Pp.212-220). DOI: } & \end{array}$ https://doi.org/10.1016/j.jbusres.2016.10.032

Hartley, Jean; Alford, John; Douglas, Scott. (2016). Towards an empirical research agenda for public value theory. Public Management Review, 19(5). Reino Unido. (Pp.670-685). DOI: https://doi.org/10.1080/14719037.2016.1192166

Hofmann, Sara; Sæbø, Øystein; Braccini, Alessio; Za, Stefano. (2019). The public sector's roles in the sharing economy and the implications for public values. Government Information

Quarterly, 36(4). Reino Unido. (Pp.1-12). DOI: https://doi.org/10.1016/i.giq.2019.101399 Jørgensen, Torben; Bozeman, Barry. (2007). Public Values: An Inventory. Administration and Society, $\quad 39(3)$. Estados Unidos. (Pp.354-381). DOI: https://doi.org/10.1177/0095399707300703

Mair, Johanna. (2006). Introduction to Part II — Exploring the Intentions and Opportunities Behind Social Entrepreneurship. Social Entrepreneurship, In: Mair Johanna; Robinson Jeffrey; Hockerts Kai. (eds). Palgrave Macmillan. Inglaterra. DOI: https://doi.org/10.1057/9780230625655 6

Meynhardt, Timo. (2009). Public Value Inside: What is Public Value Creation? . International Journal of Public Administration, 32(3-4). Estados Unidos. (Pp.192-219). DOI: https://doi.org/10.1080/01900690902732632

Moore, Mark. (1995). Creating Public Value: Strategic Management in Government. Harvard University Press. Estados Unidos.

Nicholls, Alex. (2006). Social Entrepreneurship: New Models of Sustainable Social Change (primera edición). Oxford University Press. Estados Unidos.

Putnam, Robert. (2000). Bowling Alone: The Collapse and Revival of American Community (primera edición). Touchstone Books by Simon \& Schuster. Estados Unidos. 
Financiamiento para investigaciones en Innovación Social de la Academia Latinoamericana:

Retos y desafíos

Putnam, Robert; Leonardi, Robert; Nanetti, Raffaella. (1994). Making democracy work: civic traditions in modern Italy. Princeton University Press. Estados Unidos.

Reynaers, Anne-Marie., \& Graaf, Gjalt. (2014). Public Values in Public-Private Partnerships. International Journal of Public Administration, 37(2). Estados Unidos. (Pp.120-128). DOI: https://doi.org/10.1080/01900692.2013.836665

Robinson, Jeffrey. (2006). Navigating Social and Institutional Barriers to Markets: How Social Entrepreneurs Identify and Evaluate Opportunities. En Mair. Johanna; Robinson. Jeffrey; Hockers. Kai. Social Entrepreneurship. Palgrave Macmillan. Estados Unidos.

Sánchez, José (2009). El mundo después de la revolución: la física de la segunda mitad del siglo $X X$. Fronteras del Conocimiento, BBVA. España.

Sanz, Nuria. (2017). Innovación y cooperación social, científica e intelectual para el Desarrollo Sostenible 2030: una apuesta desde la UNESCO México. Innovación para el Desarrollo Sostenible. Guanajuato, México. https://core.ac.uk/download/pdf/195282992.pdf

Stoker, Gerry. (2006). Public Value Management: A New Narrative for Networked Governance?. The American Review of Public Administration, 36(1). Reino Unido. (Pp.41-57). DOI: https://doi.org/10.1177/0275074005282583

UNESCO. (1999). Declaración sobre la ciencia y el uso del saber científico. Science for the Twenty-First Century. A new Commitment. World Conference on Science. Budapest, Hungría http://www.unesco.org/science/wcs/esp/declaracion_s.htm

Van Wart, Montgomery. (1998). Changing Public Sector Values. Routledge. Reino Unido.

Williams, Brian; Seong-Cheol, Kang; Johnson, Johnson (2015). Contamination as the Dark Side of $\mathrm{Co}$-Production: Public value failures in co-production processes. Public Management $\begin{array}{llll}\text { Review, } & \text { 18(5). Reino Unido. (Pp.695-717). DOI: }\end{array}$ https://doi.org/10.1080/14719037.2015.1111660 